\title{
Influence of a water physical rehabilitation program on the hemodynamic parameters in breast cancer survivors
}

\author{
DOI: https://doi.org/10.5114/pq.2019.84267
}

\author{
Tetiana Odynets', Yuriy Briskin', levgeniia Zakharina ${ }^{2}$, Anzhelika Yefremova ${ }^{3}$ \\ ${ }^{1}$ Department of Theory of Sport and Physical Culture, Lviv State University of Physical Culture, Lviv, Ukraine \\ 2 Department of Theoretical Foundations of Physical and Adaptive Education, Classic Private University, \\ Zaporizhzhya, Ukraine \\ ${ }^{3}$ Department of Physical Education and Sport, Ukrainian State University of Railway Transport, Kharkiv, Ukraine
}

\begin{abstract}
Introduction. Numerous women experience fatigue, arrhythmias, heart failure, ischemic heart disease, and pulmonary side effects induced by breast cancer treatment. The aim of the study was to analyse the changes in hemodynamic parameters in breast cancer survivors under the influence of a water physical rehabilitation program.

Methods. Overall, 34 women after breast cancer treatment completed a water physical rehabilitation program (group A) and 34 completed a Pilates program (group B). In both groups, the participants performed the same number of sessions 3 times a week for 3 months. The study was conducted during outpatient rehabilitation. Hemodynamic parameters were evaluated with impedance cardiography.

Results. Significant improvement was observed in both groups but it was more significant in group A. The actual value of cardiac output was higher in group A compared with group B by $0.64 \mathrm{l} / \mathrm{min}(p<0.01)$, left ventricular power by $0.49 \mathrm{~W}(p<0.01)$, and left ventricular work by $0.54 \mathrm{gm}-\mathrm{m} /$ beat $(p<0.01)$.

Conclusions. The water program resulted in more significant improvements in cardiac output, stroke index, systemic vascular resistance, left ventricular work, and left ventricular power compared with the Pilates program during outpatient rehabilitation in women after breast cancer treatment.

Key words: cardiovascular system, breast cancer, physical therapye
\end{abstract}

\section{Introduction}

Modern approaches used in breast cancer treatment have resulted in increasing survival rates. However, the incidence of significant adverse events such as cardiotoxicity or cardiovascular complications remains high [1-4]. Numerous women experience fatigue, arrhythmias, heart failure, ischemic heart disease, and pulmonary side effects induced by breast cancer treatment $[5,6]$.

Cardiovascular disease is the most common cause of mortality in patients who have survived a cancer [7]. Latest studies [8-11] have shown high prevalence of cardiovascular side effects, which results in a need for constant monitoring in breast cancer survivors.

Taking into consideration the high frequency of cardiovascular risk factors and deterioration of physical functioning, more specific attention is required in breast cancer survivors during the physical intervention.

An increasing number of studies [12-15] show that physical exercises have a potential beneficial impact on the modulation of cardiovascular risk factors, prevention of cardiac dysfunction, and improvement in physical condition of patients during and after breast cancer treatment. Some studies suggested that combined exercises (aerobic and resistance) led to significant increase in cardiorespiratory fitness, others showed the importance of using aerobic and stretching exercises to improve cardiopulmonary parameters. Only few studies reported the application of water exercises to eliminate or reduce the manifestations of lymphostasis [16-18], upper extremity impairments [19], pain [20], or cancer-related fatigue [21]. However, they did not include criteria for the selection and application of adequate means, methodological recommendations for exercises that would individualize the process of physical therapy in breast cancer survivors.

Nevertheless, it is important to emphasize that the exercise modality, frequency, duration, and intensity must be specialized for women in compliance with their physical activity level.

With the consideration of these issues, the purpose of this study was to analyse the changes in hemodynamic parameters in breast cancer survivors under the influence of water physical rehabilitation program.

\section{Subjects and methods}

Overall, 75 participants were tested for eligibility (the process of recruitment, screening eligibility, and drop-outs are shown in Figure 1), of which 4 women were excluded. A total of 68 patients who completed the study were analysed. The study groups were homogeneous by all parameters at baseline. Treatment information and demographic characteristics of the sample are presented in Table 1. The research was conducted among women aged $50-60$ years with I-II cancer stage, not more than 6 months after surgery. The focus on women aged 50-60 years was used because the incidence of breast cancer is the highest in this age category. Women with III cancer stage, metastases, congestive heart failure, and

Correspondence address: Tetiana Odynets, Department of Theory of Sport and Physical Culture, Lviv State University of Physical Culture, 79000, 11, Kostiushko Str., Lviv, Ukraine, e-mail: tatyana01121985@gmail.com 


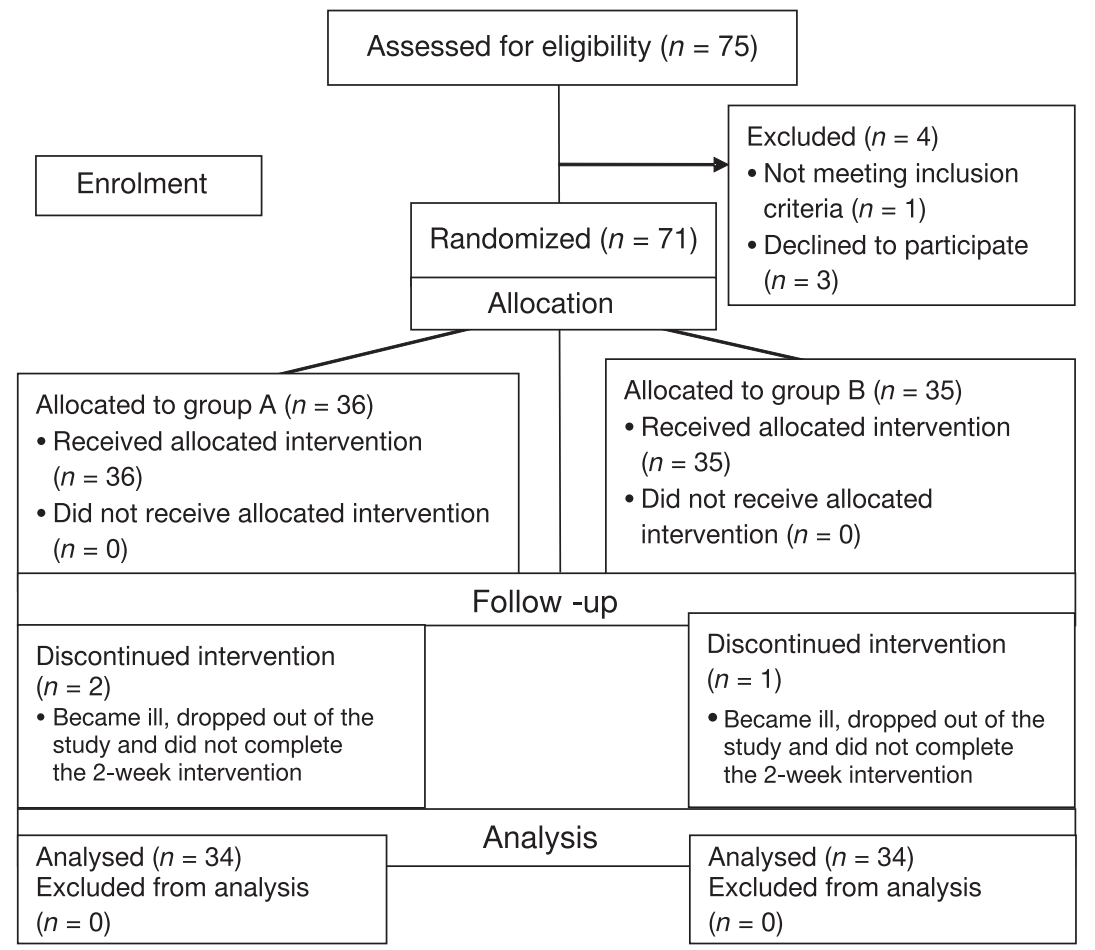

Figure 1. The CONSORT flow diagram

Table 1. Demographic and treatment-related characteristics of the participants

\begin{tabular}{|c|c|c|c|}
\hline Characteristics & $\begin{array}{l}\text { Water exercise group } \\
\qquad(n=34)\end{array}$ & $\begin{array}{l}\text { Pilates group } \\
\qquad(n=34)\end{array}$ & $p$ \\
\hline Age, $M \pm m$ & $57.44 \pm 2.16$ & $57.99 \pm 2.24$ & $>0.05$ \\
\hline \multicolumn{4}{|l|}{ Race } \\
\hline White, $n(\%)$ & $33(97 \%)$ & 32 (94\%) & $>0.05$ \\
\hline Black, $n(\%)$ & $1(3 \%)$ & $2(6 \%)$ & $>0.05$ \\
\hline Married / committed relationship, $n$ (\%) & $28(82 \%)$ & 27 (79\%) & $>0.05$ \\
\hline High school graduate, $n(\%)$ & $13(38 \%)$ & $12(35 \%)$ & $>0.05$ \\
\hline College graduate, $n(\%)$ & $19(56 \%)$ & $20(59 \%)$ & $>0.05$ \\
\hline Post-graduate, $n(\%)$ & $2(6 \%)$ & $2(6 \%)$ & $>0.05$ \\
\hline Body mass index, $\mathrm{kg} / \mathrm{m}^{2}, M \pm m$ & $25.92 \pm 0.42$ & $26.01 \pm 0.81$ & $>0.05$ \\
\hline \multicolumn{4}{|l|}{ Treatment } \\
\hline Surgery type: mastectomy by Madden, $n(\%)$ & $34(100 \%)$ & $34(100 \%)$ & $>0.05$ \\
\hline \multicolumn{4}{|l|}{ Adjuvant therapy } \\
\hline Radiotherapy, $n(\%)$ & $30(88 \%)$ & $31(91 \%)$ & $>0.05$ \\
\hline Chemotherapy, $n$ (\%) & $4(12 \%)$ & $3(9 \%)$ & $>0.05$ \\
\hline Time after surgery, months & $5.23 \pm 0.32$ & $5.11 \pm 0.42$ & $>0.05$ \\
\hline \multicolumn{4}{|l|}{ Cancer stage } \\
\hline $\mathrm{I}, n(\%)$ & $12(35 \%)$ & $13(38 \%)$ & $>0.05$ \\
\hline II, $n(\%)$ & $22(65 \%)$ & $21(62 \%)$ & $>0.05$ \\
\hline \multicolumn{4}{|l|}{ Degree of lymphedema } \\
\hline $1, n(\%)$ & $5(15 \%)$ & $6(17 \%)$ & $>0.05$ \\
\hline $2, n(\%)$ & $19(56 \%)$ & 19 (56\%) & $>0.05$ \\
\hline $3, n(\%)$ & $10(29 \%)$ & $9(27 \%)$ & $>0.05$ \\
\hline
\end{tabular}


metastatic breast cancer were excluded from the current investigation.

Women after breast cancer treatment participated in a 12-week water physical rehabilitation program (group A, $n=34$ ) and Pilates program (group $B, n=34$ ). They were randomized with the use of sequentially numbered, opaque, sealed envelopes. In both groups, the patients performed the same number of sessions 3 times a week for 3 months.

The proposed water rehabilitation program for 50-60-yearold women after breast cancer treatment included a differential selection of means for overcoming cardiovascular complications, with the consideration of the patients' motor function status. In order to regulate the motor function status of women during the implementation of the water program, 3 motion activity modes were applied - dose-sparing, training, and conditional regimens. There were defined individual tasks and means of their implementation, as well as methodological features in each regimen.

The individualization of session intensity and length depended on the participants' cardiovascular functional status. Women with a low level of cardiovascular functional status performed exercises with the intensity of $45-50 \%$ of heart rate reserve, lasting $50 \mathrm{~min}$; those below the average level practised with $50-55 \%$ of heart rate reserve, for $55 \mathrm{~min}$; in subjects with an average level, the intensity was $55-60 \%$ of heart rate, duration of 60 min. Exercises with low intensity and coordinating complexity were used in the dose-sparing motor activity, avoiding exercises with tension, rapid movement, or high amplitude. Special pool equipment was applied for performing strength exercises, like padded bar float, noodles, fitness bar bells. Exercises like sidestroke, deep breathing, and flotation were performed to improve water adaptation and to reduce water-repellency, as well as to ensure the participants' comfort in moving around the pool. With regard to tasks of the motor activity regimen, the program involved breathing exercises, gliding, active and passive exercises, different swimming styles, exercises for relaxation and stretching of the muscles.

The difficulty of the session content increased with the rising coordination complexity of exercises, number of means used, and the duration or intensity in the training regimen of motor activity. The content of the conditional regimen was gradually complicated with the rising coordination complexity of exercises, their intensity and duration, and additional equipment.

The Pilates program included active and passive stretching, exercises for good posture, and resistive exercises, with their intensity related to the cardiovascular functional status. Resistance and flexibility exercises targeted at all major muscle groups.

The assessments of hemodynamic parameters were undertaken twice: at baseline and after the 12-week outpatient rehabilitation. Impedance cardiography indicators were evaluated with the electrocardiographic complex KARDIOLAB (Scientific and Technological Centre of Radio-Electronic Medical Equipment and Technologies XAI-Medica of the National Aerospace University, Kharkiv, Ukraine, registration certificate number $6037 / 2007$, conformity certificate number UAMI/2p-2765-2009). The following indicators were assessed:

1. stroke volume $(\mathrm{ml} /$ beat): amount of blood pumped by the left ventricle at each heartbeat;

2. cardiac output ( $(/ \mathrm{min})$ : amount of blood pumped by the left ventricle per minute;

3. stroke index $\left(\mathrm{ml} / \mathrm{beat} / \mathrm{m}^{2}\right)$ : stroke volume normalized for body surface area;
4. systemic vascular resistance $\left(\right.$ dyne $\left.\cdot \mathrm{s} \cdot \mathrm{cm}^{5}\right)$ : the resistance to the flow of blood in the vasculature;

5. left ventricular work (gm-m/beat): the amount of work that the left ventricle must perform to pump blood each minute;

6 . left ventricular power (W): left ventricular work performed per time unit [22].

All indicators of impedance cardiography were equivalent in the study groups at the beginning of the rehabilitation. Thus, the groups were homogeneous at the start of the study. The analyses of impedance cardiography indicators were performed by using the Statistica software. All variables were analysed for normality with the Shapiro-Wilk test. According to the Shapiro-Wilk test, the data tested were normally distributed. To analyse the differences between the studied groups, the independent samples $t$-test was applied. The dependent samples $t$-test served to describe intra-group pre- and postintervention changes. Impedance cardiography parameters were presented as means and standard errors of mean.

\section{Ethical approval}

The research related to human use has complied with all the relevant national regulations and institutional policies, has followed the tenets of the Declaration of Helsinki, and has been approved by the Ethics Committee of Khortytsia National Academy, Zaporizhzhya, Ukraine (No. 2017/12-11).

\section{Informed consent}

Informed consent has been obtained from all individuals included in this study.

\section{Results}

Repeated examination was conducted to assess the efficiency of the proposed rehabilitation programs for women after breast cancer surgery. The study revealed a significant impact of the applied water physical rehabilitation program and Pilates program on the patients' cardiovascular function. Statistically significant improvements were identified in both groups $(p<0.05)$.

The dynamics of hemodynamic parameters in both group participants during the 12-week outpatient rehabilitation is shown in Table 2.

The results suggest that all indicators of the cardiovascular function improved significantly after the 12-week water program in group $A$. This particularly refers to the actual stroke volume, which improved by $7.05 \mathrm{ml} /$ beat $(p<0.001)$; to stroke index, which improved by $0.41 \mathrm{ml} / \mathrm{beat} / \mathrm{m}^{2}(p<0.001)$; to cardiac output, which improved by $0.69 \mathrm{l} / \mathrm{min}(p<0.001)$; to left ventricular work, which improved by $0.68 \mathrm{gm}-\mathrm{m} /$ beat $(p<0.001)$; and to left ventricular power, which improved by $0.38 \mathrm{~W}(p<0.001)$. Also, a significant decrease in systemic vascular resistance by 343.33 dyne $\cdot s \cdot \mathrm{cm}^{5}(p<0.001)$ should be noted.

Regarding the hemodynamic parameters of group $\mathrm{B}$, there were no statistically significant changes during the 12-week rehabilitation period.

Comparing the results of cardiovascular function after the 12-week appropriate outpatient programs, it was found that in women of group A compared with those of group B, the actual value of cardiac output was higher by $0.64 \mathrm{l} / \mathrm{min}(p<$ $0.01)$, that of left ventricular power by $0.49 \mathrm{~W}(p<0.01)$, and that of left ventricular work by $0.54 \mathrm{gm}-\mathrm{m} /$ beat $(p<0.01)$. The actual value of systemic vascular resistance was statistically lower by 265.77 dyne $\cdot \mathrm{s} \cdot \mathrm{cm}^{5}(p<0.05)$ in group $A$ than in group B. 
Table 2. Dynamics of hemodynamic parameters in groups A and B during the 12-week outpatient rehabilitation

\begin{tabular}{|c|c|c|c|c|c|c|c|}
\hline \multirow{2}{*}{\multicolumn{2}{|c|}{ Indicator }} & \multicolumn{2}{|c|}{ Group A $(n=34)$} & \multirow{2}{*}{$p$} & \multicolumn{2}{|c|}{ Group B $(n=34)$} & \multirow{2}{*}{$p$} \\
\hline & & Beginning & 12 weeks & & Beginning & 12 weeks & \\
\hline \multirow[b]{2}{*}{ Stroke volume (ml/beat) } & actual & $47.18 \pm 2.25$ & $54.23 \pm 1.78$ & $<0.001$ & $47.53 \pm 2.00$ & $50.26 \pm 2.11$ & $>0.05$ \\
\hline & $\begin{array}{l}\text { \% of pre- } \\
\text { dicted }\end{array}$ & $75.83 \pm 2.84$ & $88.47 \pm 3.03$ & $<0.001$ & $76.43 \pm 3.27$ & $81.00 \pm 3.73$ & $>0.05$ \\
\hline \multicolumn{2}{|l|}{ Cardiac output (I/min) } & $3.53 \pm 0.14$ & $4.22 \pm 0.17^{\star \star}$ & $<0.001$ & $3.44 \pm 0.13$ & $3.58 \pm 0.13$ & $>0.05$ \\
\hline \multicolumn{2}{|l|}{ Stroke index $\left(\mathrm{ml} / \mathrm{beat} / \mathrm{m}^{2}\right)$} & $1.99 \pm 0.07$ & $2.40 \pm 0.10^{*}$ & $<0.001$ & $2.03 \pm 0.09$ & $2.11 \pm 0.09$ & $>0.05$ \\
\hline \multirow{2}{*}{$\begin{array}{l}\text { Systemic vascular resistance } \\
\left(\text { dyne } \cdot \mathrm{s} \cdot \mathrm{cm}^{5}\right)\end{array}$} & actual & $2080.03 \pm 84.26$ & $1736.70 \pm 76.27^{\star}$ & $<0.001$ & $2097.60 \pm 82.36$ & $2002.47 \pm 87.98$ & $>0.05$ \\
\hline & $\begin{array}{l}\% \text { of pre- } \\
\text { dicted }\end{array}$ & $129.87 \pm 5.08$ & $108.23 \pm 4.62^{*}$ & $<0.001$ & $128.77 \pm 5.48$ & $122.57 \pm 5.39$ & $>0.05$ \\
\hline \multirow[b]{2}{*}{ Left ventricular power (W) } & actual & $2.27 \pm 0.09$ & $2.65 \pm 0.09^{\star * \star}$ & $<0.001$ & $2.16 \pm 0.08$ & $2.16 \pm 0.07$ & $>0.05$ \\
\hline & $\begin{array}{l}\% \text { of pre- } \\
\text { dicted }\end{array}$ & $85.60 \pm 2.77$ & $100.80 \pm 3.60^{\star \star *}$ & $<0.001$ & $82.13 \pm 3.12$ & $82.67 \pm 3.13$ & $>0.05$ \\
\hline \multirow{2}{*}{$\begin{array}{l}\text { Left ventricular work } \\
\text { (gm-m/beat) }\end{array}$} & actual & $4.27 \pm 0.13$ & $4.95 \pm 0.15^{\star *}$ & $<0.001$ & $4.38 \pm 0.12$ & $4.41 \pm 0.12$ & $>0.05$ \\
\hline & $\begin{array}{l}\% \text { of pre- } \\
\text { dicted }\end{array}$ & $76.42 \pm 2.11$ & $88.73 \pm 2.71^{\star *}$ & $<0.001$ & $77.44 \pm 2.12$ & $77.94 \pm 2.12$ & $>0.05$ \\
\hline
\end{tabular}

${ }^{*} p<0.05,{ }^{* *} p<0.01,{ }^{* * *} p<0.001$ for comparison of the 12-week intervention results between groups $\mathrm{A}$ and $\mathrm{B}$

\section{Discussion}

The performed research has presented a significant impact of outpatient rehabilitation on improving the functional status of the cardiovascular system in breast cancer survivors. The positive results might be associated not only with the rational scheme of the rehabilitation process, but also with the physiological influence of the aquatic environment.

The hydrostatic pressure of the water medium contributes to the emergence of compensatory reactions from the cardiovascular system in the form of pulse deceleration and decreased blood circulation, which might lead to the economization of functions $[17,19]$. Most studies confirm the positive influence of aerobic and water exercises on physical fitness, body composition, and blood pressure of older adults [23-25].

The water physical rehabilitation program resulted in significant improvements in the stroke volume, cardiac output, stroke index, systemic vascular resistance, left ventricular work, and left ventricular power in women during outpatient rehabilitation.

A detailed analysis of literary sources [4, 9, 10, 13, 23, 24], including randomized clinical trials, pilot-analysis, and metaanalysis, showed that women after breast cancer treatment experienced radiotherapy- and chemotherapy-induced cardiovascular system adverse events, fatigue, and cardiotoxicity. Some papers considered aerobic physical activity [26] and water physical therapy $[20,27,28]$ for pain and lymphedema reduction in post-mastectomy women, but they did not examine the effect of water exercises on the improvement of hemodynamic parameters in breast cancer survivors.

The proposed water program applied load differentiation in the exercise intensity and duration depending on the patients' cardiovascular system functional status. Three motion activity modes were applied for the gradual expansion of the functional capabilities of the women's cardiovascular system. Significant improvement was observed in both groups but it was more significant in group A. The more positive effect of the water program compared with the Pilates program may be due to the properties of the aquatic environ- ment that helped improve the parameters of central hemodynamics, in particular the hydrostatic pressure and lack of static tension, in combination with deep rhythmic breathing and dynamic muscle contraction. All of the above created favourable conditions for facilitated blood circulation and increased functionality of the cardiovascular system.

\section{Limitations}

Despite these strengths, the current research also has some important weaknesses. Firstly, our investigation involved a limited number of patients. Secondly, the received results cannot be applied to all breast cancer survivors because of the specific experiment conditions and cultural background.

\section{Conclusions}

Significant improvement was observed in both groups but it was more significant in group A. The water physical rehabilitation program resulted in more significant improvements in cardiac output, stroke index, systemic vascular resistance, left ventricular work, and left ventricular power compared with the Pilates program during outpatient rehabilitation in women after breast cancer therapy.

\section{Disclosure statement}

No author has any financial interest or received any financial benefit from this research.

\section{Conflict of interest}

The authors state no conflict of interest.

\section{References}

1. Martel S, Maurer C, Lambertini M, Pondé N, De Azambuja E. Breast cancer treatment-induced cardiotoxicity. Expert Opin Drug Saf. 2017;16(9):1021-1038; doi: 10.1080/14740338.2017.1351541.

2. Hooning MJ, Botma A, Aleman BM, Baaijens MH, Bartelink H, Klijn JG, et al. Long-term risk of cardiovascular 
disease in 10-year survivors of breast cancer. J Natl Cancer Inst. 2007;99(5):365-375; doi: 10.1093/jnci/djk064.

3. Rygiel K. Cardiotoxic effects of radiotherapy and strategies to reduce them in patients with breast cancer: an overview. J Cancer Res Ther. 2017;13(2):186-192; doi: 10.4103/0973-1482.187303.

4. Cueva JF, Antolín S, Calvo L, Fernández I, Ramos M, de Paz L, et al. Galician consensus on management of cardiotoxicity in breast cancer: risk factors, prevention, and early intervention. Clin Transl Oncol. 2017;19(9): 1067-1078; doi: 10.1007/s12094-017-1648-8.

5. Yu AF, Jones LW. Breast cancer treatment-associated cardiovascular toxicity and effects of exercise countermeasures. Cardiooncology. 2016;2; doi: 10.1186/s40959016-0011-5.

6. Fabian C. Prevention and treatment of cardiac dysfunction in breast cancer survivors. Adv Exp Med Biol. 2015; 862:213-230; doi: 10.1007/978-3-319-16366-6 14.

7. Zagar TM, Cardinale DM, Marks LB. Breast cancer therapyassociated cardiovascular disease. Nat Rev Clin Oncol. 2016;13(3):172-184; doi: 10.1038/nrclinonc.2015.171.

8. Kwan ML, Sternfeld B, Ergas IJ, Timperi AW, Roh JM, Hong CC, et al. Change in physical activity during active treatment in a prospective study of breast cancer survivors. Breast Cancer Res Treat. 2012;131(2):679-690; doi: 10.1007/s10549-011-1788-4.

9. Anulika Aweto $\mathrm{H}$, Akinbo SR, Olawale OA. Effects of combined aerobic and stretching exercises on the cardiopulmonary parameters of premenopausal and postmenopausal breast cancer survivors. Nig Q J Hosp Med. 2015;25(3):177-183.

10. Lipsett A, Barrett S, Haruna F, Mustian K, O’Donovan A. The impact of exercise during adjuvant radiotherapy for breast cancer on fatigue and quality of life: a systematic review and meta-analysis. Breast. 2017;32:144-155; doi: 10.1016/j.breast.2017.02.002.

11. Odinets T, Briskin Y. Impact of personality-oriented programs of physical rehabilitation on the heart rate variability in women with post-mastectomy syndrome. Fizjoterapia/Physiotherapy. 2016;24(2):4-8; doi: 10.1515/ physio-2016-0007.

12. Adraskela K, Veisaki E, Koutsilieris M, Philippou A. Physical exercise positively influences breast cancer evolution. Clin Breast Cancer. 2017;17(6):408-417; doi: 10.1016/j. clbc.2017.05.003.

13. Scott JM, Adams SC, Koelwyn GJ, Jones LW. Cardiovascular late effects and exercise treatment in breast cancer: current evidence and future directions. Can J Cardiol. 2016;32(7):881-890; doi: 10.1016/j.cjca.2016.03.014.

14. Casla S, López-Tarruella S, Jerez Y, Marquez-Rodas I, Galvão DA, Newton RU, et al. Supervised physical exercise improves VO2max, quality of life, and health in early stage breast cancer patients: a randomized controlled trial. Breast Cancer Res Treat. 2015;153(2):371382; doi: 10.1007/s10549-015-3541-x.

15. Rahmani H, Shahriary A, Sheikhi MA, Ebadi A, Davoodzadeh $\mathrm{H}$. Applications of cardiotoxicity in breast cancer: a meta-analysis. Panminerva Med. 2017;59(1):90-96; doi: 10.23736/S0031-0808.16.03201-8.

16. Lindquist H, Enblom A, Dunberger G, Nyberg T, Bergmark K. Water exercise compared to land exercise or standard care in female cancer survivors with secondary lymphedema. Lymphology. 2015;48(2):64-79.

17. Tidhar D, Katz-Leurer M. Aqua lymphatic therapy in women who suffer from breast cancer treatment-related lymphedema: a randomized controlled study. Sup- port Care Cancer. 2010;18(3):383-392; doi: 10.1007/ s00520-009-0669-4.

18. Ambroza C, Geigle PR. Aquatic exercise as a management tool for breast cancer-related lymphedema. Top Geriatr Rehabil. 2010;26(2):120-127; doi: 10.1097/TGR. 0b013e3181dfd91f.

19. Thein JM, Brody LT. Aquatic-based rehabilitation and training for the shoulder. J Athl Train. 2000;35(3):382389.

20. Cantarero-Villanueva I, Fernández-Lao C, Fernándezde-Las-Peñas C, López-Barajas IB, Del-Moral-Ávila R, de la-Llave-Rincón Al, et al. Effectiveness of water physical therapy on pain, pressure pain sensitivity, and myofascial trigger points in breast cancer survivors: a randomized, controlled clinical trial. Pain Med. 2012;13(11): 1509-1519; doi: 10.1111/j.1526-4637.2012.01481.x.

21. Cantarero-Villanueva I, Fernández-Lao C, Cuesta-Vargas Al, Del Moral-Avila R, Fernández-de-Las-Peñas C, Arroyo-Morales $\mathrm{M}$. The effectiveness of a deep water aquatic exercise program in cancer-related fatigue in breast cancer survivors: a randomized controlled trial. Arch Phys Med Rehabil. 2013;94(2):221-230; doi: 10.1016/ j.apmr.2012.09.008.

22. Cybulski G. Ambulatory impedance cardiography: the systems and their applications. Berlin: Springer; 2011.

23. De Luca V, Minganti C, Borrione P, Grazioli E, Cerulli C, Guerra E, et al. Effects of concurrent aerobic and strength training on breast cancer survivors: a pilot study. Public Health. 2016;136:126-132; doi: 10.1016/j.puhe.2016. 03.028 .

24. Dolan LB, Gelmon K, Courneya KS, Mackey JR, Segal RJ, Lane $\mathrm{K}$, et al. Hemoglobin and aerobic fitness changes with supervised exercise training in breast cancer patients receiving chemotherapy. Cancer Epidemiol Biomarkers Prev. 2010;19(11):2826-2832; doi: 10.1158/10559965.EPI-10-0521.

25. Pereira Neiva $H$, Brandão Faíl L, Izquierdo $M$, Marques MC, Marinho DA. The effect of 12 weeks of water-aerobics on health status and physical fitness: an ecological approach. PLoS One. 2018;13(5):e0198319; doi: 10.1371/journal.pone.0198319.

26. Włoch A, Bocian A, Biskup M, Krupnik S, Opuchlik A, Ridan T, et al. Effectiveness of specific types of structured physical activities in the rehabilitation of post-mastectomy women: a systematic review. Medical Studies/Studia Medyczne. 2018;34(1):86-92; doi: 10.5114/ms.2018. 74826.

27. Ergin G, Karadibak D, Sener HO, Gurpinar B. Effects of aqua-lymphatic therapy on lower extremity lymphedema: a randomized controlled study. Lymphat Res Biol. 2017;15(3):284-291; doi: 10.1089/Irb.2017.0017.

28. Odynets T, Briskin Y, Sydorko O, Tyshchenko V, Putrov S. Effectiveness of individualized physical rehabilitation programs on post-mastectomy pain in breast cancer survivors. Physiother Quart. 2018;26(3):1-5; doi: 10.5114/ pq.2018.78377. 